\title{
Symmetry Identification Using Partial Surface Matching and Tilt Correction in 3D Brain Images
}

\author{
Xin Liu, Celina Imielinska, Andrew Laine, E. Sander Connolly, Anthony L. D'Ambrosio
}

\begin{abstract}
We propose a novel method to automatically compute the symmetry plane and correct the 3D orientation of patient brain images. Many images of the brain are clinically unreadable because of the misalignment of the patient's head in the scanner. We proposed an algorithm that represents the brain volume as a re-parameterized surface point cloud where each location has been parameterized by its elevation (latitude), azimuth (longitude) and radius. The removal of the interior contents of the brain makes this approach perform robustly in the presence of the brain pathologies e.g. tumor, stroke and bleed. Thus, we decompose the symmetry plane computation problem into a surface matching routine. The search for the best matching surface is implemented in a multi-resolution paradigm so as to decrease computational time considerably. Spatial affine transform then is performed to rotate the $3 D$ brain images and align them within the coordinate system of the scanner. The corrected brain volume is re-sliced such that each planar image represents the brain at the same axial level.
\end{abstract}

\section{INTRODUCTION}

Human brain exhibits high level of bilateral symmetry, although it is not perfectly symmetrical. Detection and computation of symmetry plane in the brain has many applications. Symmetry is used by clinical experts to detect qualitatively asymmetric pattern indicating a wide range of pathologies. Similarly computer aided systems are used to quantify asymmetry and to automatically generate hints for clinicians. Since neuroradiologists use routinely symmetry in their assessment of brain images, the misalignment of the patient's head in the scanner often leads to false clinical interpretation of the patients scans. Likewise, for computer program to correctly assess the pathological asymmetries it is crucial that the neural scans are not tilted but correctly aligned and oriented within the coordinate system of the scanner. However, the tilt of the head that is observed in practice quite often, not always can be controlled. It may be

Xin Liu is PhD Student in Department of Biomedical Informatics, Columbia University. New York, NY 10032, USA. Phone: 212-342-1632; Fax: 212-342-1647; Email: x12104@columbia.edu .

Celina Imielinska, is with Department of Biomedical Informatics, and Department of Computer Science, Columbia University. New York, NY 10032.USA; Email: ci42@columbia.edu.

Andrew Laine, is with Department of Biomedical Engineering, Columbia University . New York, NY10027 USA; Email: 1 laine@columbia.edu

E. Sander Connolly, is with Neurosurgery Department, Columbia University, New York, NY 10032.USA; Email: esc5@columbia.edu

Anthony L. D'Ambrosio is with Neurosurgery Department, Columbia University, New York, NY 10032.USA; Email: ad504@columbia.edu caused by the immobility of the patient, inexperience of the technician, or the imaging device itself. This makes radiological slices of the brain images no longer homologous within the same coronal or axial level. Thus, both assessment either by a clinical expert and/or automatic system based on symmetry analysis, like one similar to the Relative Difference Map (RDM) quantification [3], require first the brain to be re-aligned within the scanner coordinate system.

The paper is organized as follows. In section 2, we review the existing methods. Our algorithm is described and discussed in section 3. Finally, the results are presented in section 4 and we conclude our work and point out future directions in section 5 .

\section{BACKGROUND}

There are two major approaches to solve the problem of computing the symmetry plane in brain images. One is the $2 \mathrm{D}$ based method, and the other is the volume based method. The number of reported methods to compute brain symmetry, in $3 \mathrm{D}$ volume is considerably smaller than those in $2 \mathrm{D}$. The planar method that is applied to 2D radiological brain slices, may not always be extendable to 3D cases. For example, Brummer [4] proposes a method of using the Hough transform to identify cerebral inter-hemisphereic fissure. Marais [9] extracts the fissure using snakes, and uses an orthogonal regression from a set of control points. The method presented by Liu [7], estimates 2D mid-sagittal axis for each coronal or axial slice, and then computes a 3D plane from set of these lines. Because these methods process brain volume slice-by-slice, the global symmetry of the whole brain is not captured. In case where the head is tilted along the axis from posterior to anterior, a structure displayed in the same axial slice will not reside in the same plane and the symmetry axes computed independently in each slice will produce flawed final result. In 3D approaches, the plane that maximizes the bilateral symmetry is captured. Prima et al [2] computes local similarity measures between two sides of the brain, using block matching procedure . Minovic proposed using principle axes to characterize symmetry plane [8], and , Sun [6] extended Minovic's work and developed an algorithm for finding symmetry planes of 3D objects using extended Gaussian image representation. However both methods only focused on synthesized object and may be applicable if the clinical brain images have truncated field of view. Ardekani [5], conducts iterative search on the unit sphere, in order to find the plane with respect to which the 
image exhibits maximum symmetry. These algorithms that are based on local search reduce the amount of computation, but fail on clinical brain images with gross asymmetries often caused by pathological conditions.

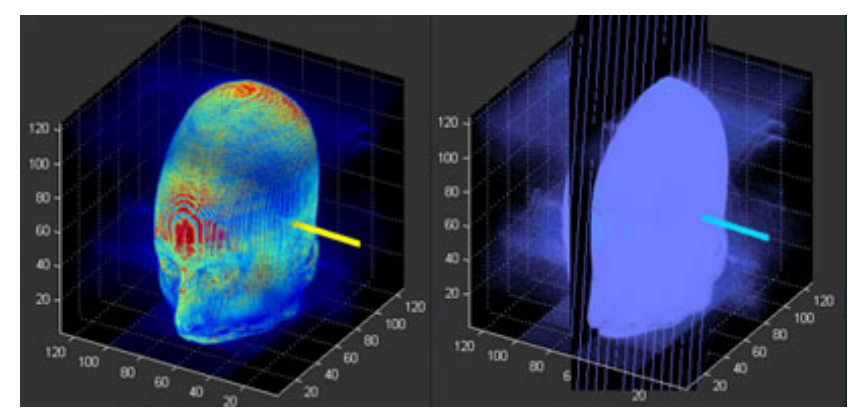

Fig.1. We use surface normal to characterize the symmetry plane in volumetric neuro-images.

In this paper propose a novel method to automatically compute the symmetry plane and correct the 3D orientation of patient brain images. Similar to most existing 3D approaches, we define the mid-sagittal plane as the one maximizes the similarity between two halves of the brain. Therefore the problem decomposes into searching over a set of possible planes to achieve the maximum of similarity measure between the original image and its reflection. When the 3-D volume is taken into account, the robustness is difficult to achieve with global criteria that is affected by the strong underling asymmetry. For most existing 3D methods, apart from their sensitivity to pathological asymmetries, another common drawback is the computational cost due to the optimization scheme when searching through the set of possible planes.

Different from those 3D approaches, we choose the surface normal to characterize the geometry of the symmetry planes in 3D Euclidian space. See Fig.1. Instead of using intensities [2] or edges [7] as the characteristic features, the similarity criterion is computed from the point cloud on the surface of the brain., regardless the contents inside. We therefore transform the $3 \mathrm{D}$ brain volume into a thin point cloud and each location on the surface has been parameterized by its elevation (latitude), azimuth (longitude) and radius. See Fig.2. The removal of the interior contents of the brain makes this approach perform robustly in the presence of the brain pathologies e.g. tumor, stroke and bleed.

After re-parameterization, we decompose the symmetry computation problem into a surface matching routine. The search for the best matching surface patches is performed utilizing a multi-resolution approach which decreases computational time considerably.

Lastly, spatial affine transform is performed to rotate the $3 \mathrm{D}$ brain images and align them within the coordinate system of the scanner. The corrected brain volume is re-sliced such that each planar image represents the brain at the same axial level. The algorithm is 3-D and is insensitive to acquisition noise, bias field and pathological asymmetries and the incomplete field of view. In the following sections, we will detail the algorithm.

\section{METHOD}

\section{A. Data representation}

Let us assume that we have a single object (the head) of interest in a volumetric dataset. We assume that patient scans do not suffer from skull or skin lesion so that the surface of the head is complete and almost symmetrical. Given the region of interest $R$ within the image $I, \delta$ is the background cutoff that separates background from the head. We assign a very small value to $\delta$ since the background intensity in most image modalities is close to zero. $X_{R}$, the characteristic function can be found as follows:

$$
X_{R}(x, y, z)=\left\{\begin{array}{cc}
1 & \text { if }(x, y, z) \in R: I(x, y, z)>\delta \\
0 & \text { otherwise }
\end{array}\right.
$$

With the integration over the whole image $I$, the volume $A$ is defined as the $0^{\text {th }}$ moment of $R[1]$.

$$
A=\iiint_{I} X_{R}(x, y, z) d x d y d z=\iiint_{R} d x d y d z
$$

Then the centroid $C=(\bar{x}, \bar{y}, \bar{z})$ is computed as[1]

$$
\begin{aligned}
& \bar{x}=\left(\iiint_{R} x d x d y d z\right) / A ; \\
& \bar{y}=\left(\iiint_{R} y d x d y d z\right) / A ; \\
& \bar{z}=\left(\iiint_{R} z d x d y d z\right) / A
\end{aligned}
$$

Although it is possible that $X_{R}$ will assign some inner structures that present low intensities to be zero, but this won't affect the correct computation of the centroid. The technique extracts the points on the surface of the brain and maps them onto spherical polar coordinates. We define $\alpha$ to be the azimuthal angle in the $x y$-plane from the $x$ axis with $0 \leq \alpha<2 \pi$, and define $\theta$ to be the polar angle from the z-axis with $-\pi / 2 \leq \theta<\pi / 2$, See Fig. 2 . We let the $\theta$ be zero at the equator, and $0 \leq \theta<\pi / 2$ for points from equator to the north pole. The algorithm allows rays $\{r(\alpha, \theta)\}$ emanating from the centroid $C$ to the boundary elements of $X_{R}$. The intersections of the ray with the furthest boundary of the region entail the sampled surface point cloud of the brain. Every point on such a point cloud $\{(x, y, z)\}$ has been uniquely mapped to a triple set $\{(\alpha, \theta, r)\}$ where the radius $r$ is the distance from a point to the centroid. $\alpha$ is also called azimuth (longitude ) and $\theta$ elevation (latitude). 


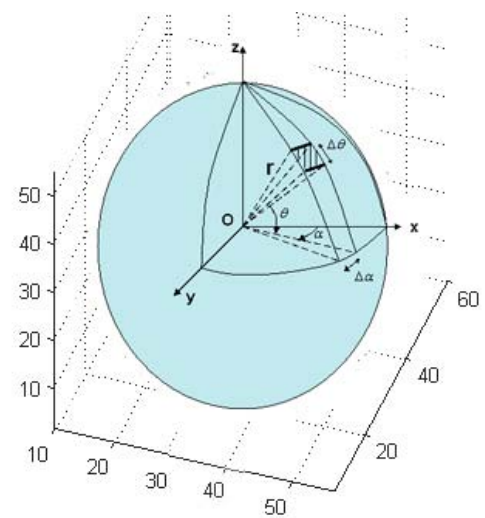

Fig.2.We represent the 3D brain volume into a surface point cloud and each location on the surface has been parameterized by its elevation (latitude), azimuth (longitude) and radius $\{(\alpha, \theta, r)\}$

\section{B. Geometry of the Mid-Sagittal Plane(MSP)}

Under Cartesian coordinates the mid-sagittal plane can be represented as

$$
a X+b Y+c Z+d=0
$$

where $N=(a, b, c)$ is the normal vector that is perpendicular to the mid-sagittal plane, and $d / \sqrt{a^{2}+b^{2}+c^{2}}$ is the perpendicular distance of the plane from the origin. Each plane is characterized by a unique set of parameters $(a, b$, c). Our aim is to find the triplet $(a, b, c)$ of a symmetry plane with respect to which the image $I$ exhibits maximum "symmetry" measure. Any parameter set $(a, b, c)$ in Cartesian coordinates has unique counterpart in spherical polar coordinates. This relationship is demonstrated as follows:

$$
\begin{aligned}
& a=R \cos \left(\theta_{N}\right) \cos \left(\alpha_{N}\right) ; \\
& b=R \cos \left(\theta_{N}\right) \sin \left(\alpha_{N}\right) ; \\
& c=R \sin \left(\theta_{N}\right) ; \\
& d=-N \cdot C
\end{aligned}
$$

Given a unit normal vector orthogonal to the symmetry plane where $R$ equals to 1 , the symmetry plane can be characterized as $N(a, b, c)=N\left(\theta_{N}, \alpha_{N}\right)$ where

$$
N\left(\theta_{N}, \alpha_{N}\right)=\left(\left(\cos \left(\theta_{N}\right) \cos \left(\alpha_{N}\right), \cos \left(\theta_{N}\right) \sin \left(\alpha_{N}\right), \sin \left(\theta_{N}\right)\right)^{T}\right.
$$

Therefore, we are essentially seeking the normal vector $N\left(\theta_{N}, \alpha_{N}\right)$ that characterizes the symmetry plane from our re-parameterized searching space until the maximum of similarity measure has been achieved.

\section{Sampling strategy and constrained search}

The rays are quantized by the discrete approximation to $\theta=k_{1} \Delta \theta, \alpha=k_{2} \Delta \alpha, k 1, k 2 \in \square$ and the step $\operatorname{size}(\Delta \theta, \Delta \alpha)$ defines the sampling resolutions. See Fig.3.

Our procedure is to select $q$ points on the point cloud and evaluate the symmetry measure with respect to the opposing $q$ points on the other side of the brain. The initial guess of the normal vector of the symmetry plane is $\theta_{N}=0, \alpha_{N}=0$, a vector directing from the centroid to the right ear. This initial guess has been iteratively refined until convergence. We define a surface patch $S\left(\left\{\theta_{i}\right\},\left\{\alpha_{i}\right\}\right)$ where its $\theta$ spans between the range
$\left[\theta_{N^{-}} k_{1} \Delta \theta, \theta_{N}+k_{1} \Delta \theta\right]$ and $\alpha$ spans between $\left[\alpha_{N}-k_{2} \Delta \alpha, \alpha_{N}+k_{2} \Delta \alpha\right]$. In our primary study, we let each surface patch $\theta$ spans 60 degrees and $\alpha$ spans 120 degrees so that it forms a roughly trapezoid shape. We set the sampling resolution to be $\Delta \theta=$ $\Delta \alpha=3^{0}$.

This gives rise to a surface patch quantized by discrete points cloud in Fig.3. This initial surface patch $S\left(\left\{\theta_{i}\right\},\left\{\alpha_{i}\right\}\right)$ should be centered around the first guess of the normal vector. We call this source surface patch (shown as the yellow cloud in Fig.3).

The search for the target surface patch (shown as the blue cloud in Fig.3) $S R\left(\left\{\theta_{i}\right\},\left\{\alpha_{i}\right\}\right)$, is the procedure to find the best matching counterpart of $S\left(\left\{\theta_{i}\right\},\left\{\alpha_{i}\right\}\right)$ on the other side of the brain. Starting from the opposite direction $v\left(\theta_{R}, \alpha_{R}\right), \theta_{R}=\theta_{N}, \alpha_{R}=\alpha_{N}+\pi$, the algorithm exams all the candidate surface patches by evaluating similarity measure in the vicinity about the vector $v\left(\theta_{R}, \alpha_{R}\right)$ within the range $\left(\theta_{R} \pm p \Delta_{\theta}, \alpha_{R} \pm p \Delta_{\alpha}\right), p \in \square$, where $\left(\Delta_{\theta}, \Delta_{\alpha}\right)$ is the sampling interval between adjacent steps. Total $4 p^{2}$ searching steps is conducted on $4 \mathrm{p} 2$ potential candidate surfaces within one iteration. $\operatorname{SR}\left(\left\{\theta_{i}\right\},\left\{\alpha_{i}\right\}\right)$ should span the same surface area as $S\left(\left\{\theta_{i}\right\},\left\{\alpha_{i}\right\}\right)$.The optimum finding of $v\left(\theta_{R}, \alpha_{R}\right)$ enters into the next iteration.

\section{Similarity measure}

For each iteration, we are searching over $4 p^{2}$ possible surfaces until we achieve the maximum of similarity measure between the source surface patch $S\left(\left\{\theta_{i}\right\},\left\{\alpha_{i}\right\}\right)$ and target surface patch $S R\left(\left\{\theta_{i}\right\},\left\{\alpha_{i}\right\}\right)$. Correlation coefficient $(C C)$ is chosen as the similarity measure. We consider all the radii of from surface $S\left(\left\{\theta_{i}\right\},\left\{\alpha_{i}\right\}\right)$ as $x_{1}, x_{2}, \ldots, x_{n}$ and all the radii from surface $S R\left(\left\{\theta_{i}\right\},\left\{\alpha_{i}\right\}\right)$ as $\mathrm{y}_{1}, \mathrm{y}_{2} \ldots, \mathrm{y}_{3}$. The correlation coefficient between $S$ and $S R$ is :

$$
C C=\frac{\frac{1}{n} \sum_{i=1}^{n}\left(x_{i}-x_{\text {mean }}\right)\left(y_{i}-y_{\text {mean }}\right)}{\frac{1}{n} \sqrt{\sum_{i=1}^{n}\left(x_{i}-x_{\text {mean }}\right)^{2} \sum_{i=1}^{n}\left(y_{i}-y_{\text {mean }}\right)^{2}}}
$$

where $-1 \leq C C \leq 1$, The $C C$ measures the strength of the linear relationship between $S$ and $S R$. Our technique seeks the highest absolute value of $C C$, the one closest to 1 , which represents the strongest correlation between source and target surface patches.

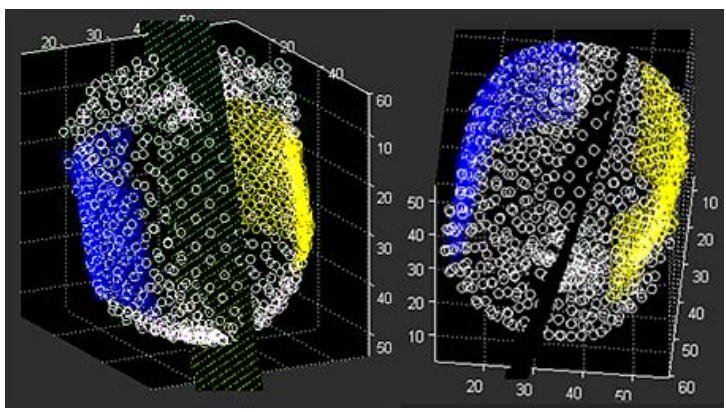

Fig.3.The algorithm extracts the head surface and re-parameterizes it into the point cloud. It then adopts multi-resolution scheme to find the best surface match between the source surface patch and the target surface path. 


\section{E. Multi-Resolution Scheme}

By adopting coarse resolution search at larger step intervals $(\Delta \theta, \Delta \alpha)$, we achieve a rough estimation of the normal vector $N\left(\theta_{N}, \alpha_{N}\right)$ at the initial runs. At iteration evolves, we reduce the search space $\left(p \Delta_{\theta}, p \Delta_{\alpha}\right)$ by applying smaller $p$, while increase the sampling resolution by shortening step size $\left(\Delta_{\theta}, \Delta_{\alpha}\right)$.Our initial search space spans $p \Delta_{\theta}=p \Delta_{\alpha}=40^{\circ}$, with sampling interval 2 degrees. We refine the search resolution by the factor of 2 at each iteration, so that at the second run, the search space spans 20 degrees at 1 degree interval, so on and so forth for subsequent iterations.

The process is repeated at finer resolution proceeding from the optimum $S R_{i}$ found by the previous iteration. The final finding of the normal vector $N\left(\theta_{N}, \alpha_{N}\right)$ can be determined by the coefficients computed from the optimum matching surfaces.

\section{$F$. Affine spatial transformation for tilt correction.}

let $R_{o}$ represent the rotation matrix as

$R_{o}=R_{\omega} R_{\beta} R_{\gamma}=$

$\left[\begin{array}{ccc}\cos (\omega) & \sin (\omega) & 0 \\ -\sin (\omega) & \cos (\omega) & 0 \\ 0 & 0 & 1\end{array}\right]\left[\begin{array}{ccc}\cos (\beta) & 0 & -\sin (\beta) \\ 0 & 1 & 0 \\ \sin (\beta) & 0 & \cos (\beta)\end{array}\right]\left[\begin{array}{ccc}1 & 0 & 0 \\ 0 & \cos (\gamma) & \sin (\gamma) \\ 0 & -\sin (\gamma) & \cos (\gamma)\end{array}\right]$

where $\omega, \beta, \gamma$ are the rotation angles with respect to the $x, y$ and $z$ axes(yaw, roll, pitch), respectively. In this case, $\omega=0, \beta=\alpha_{N}, \gamma=\theta_{N}$. Let $T$ represent the translation matrix. $V_{c}=V_{t} \cdot R_{o} \cdot T$ where $V_{t}$ is the original input volume and $V_{c}$ is the $3 \mathrm{D}$ corrected volume recentered at the centroid. The corrected brain volume is re-sliced such that each planar image represents the brain at the same axial level. We implement cubic spline interpolation to achieve better smoothness and higher precision.

\section{RESULT}

In Fig. 4, we illustrate the results on MRI volume images. We applied our method to brain MRI images from 15 patients selected from the pool of PACS (picture archiving and communication system) image database at Columbia Presbyterian Hospital. The dataset is composed of a mixture of T1-weighted MRI scans and T2-weighted MRI scans, half of which have big brain lesion and/or certain level of brain shift. Each scan is of matrix dimension $256 \times 256 \times 124$, with voxel dimension $1.01 \times 1.01 \times 2.0 \mathrm{~mm} 3$. Therefore, a total of 15 image volumes were generated. Out of 15, 14 cases were judged to be highly accurate in terms of head tilt correction and re-interpolation of the brain along the z-axis. So far, the results have only been subjectively assessed by a medical trainee, and prospective study for further quantitative validations however, is currently being designed. Initial results have shown that this method presents promising potentials to precisely capture the symmetry plane regardless of the local pathological asymmetries and acquisition noise.

\section{CONCLUSION}

This paper describes a new technique for the automatic detection of the mid-sagittal plane in arbitrarily oriented three dimensional brain images and correction the $3 \mathrm{D}$ orientation of patient brain images in a cost effective way. The algorithm is independent of the imaging modality and it is insensitive to incompleteness of the data. Unlike many of the classical symmetry-based methods, pathological asymmetries can severely degrade the computation of the symmetry plane, our method uses parameterized surface points to estimate the best similarity measure, and therefore it performs robustly in the presence of the normal/pathological asymmetries inside the brain. The search evolves at each iteration in the parameter space from the coarse level with lower resolution to the fine level with higher resolution. The use of multi-resolution paradigm dramatically reduces the computational cost while still produces satisfactory results. The future work involves more rigorous quantitative evaluation study and examination of its sensitivities to errors in surface extraction.

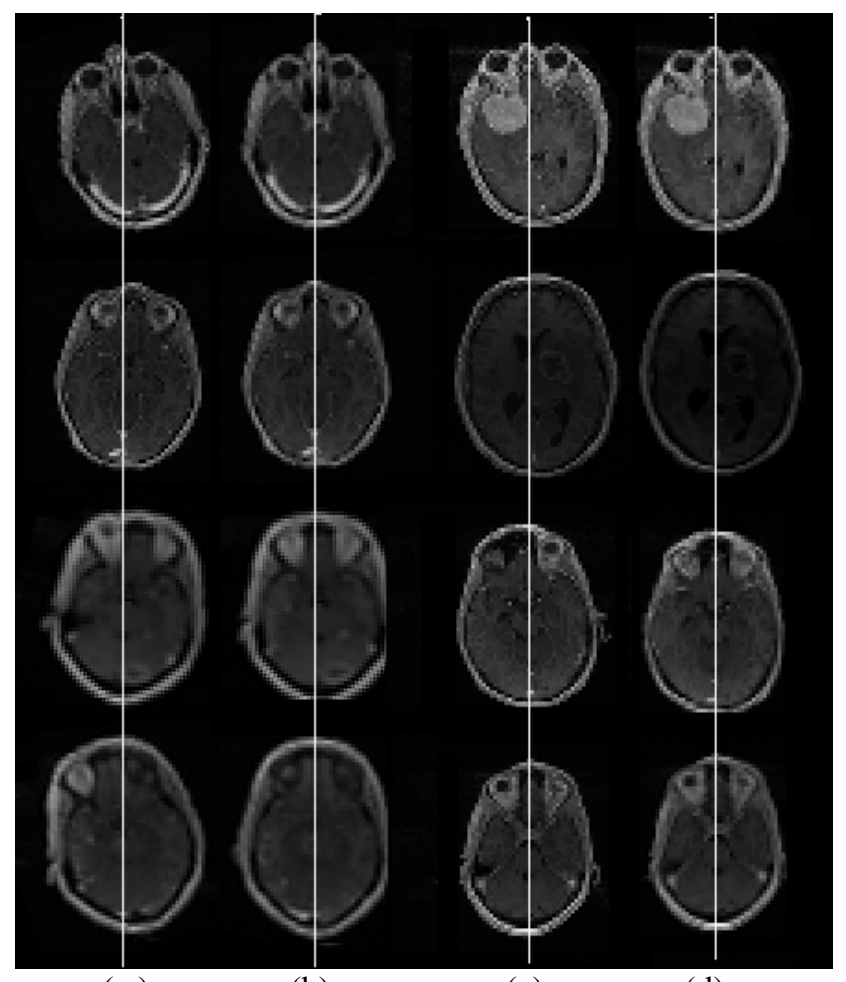

(a)

(b)

(c)

(d)

Fig.4. Results of MRI scan from 8 patients (out of 15 in total )(a) (c) columns show original potentially misaligned MRI scans; (b)(d) columns represent corresponding corrected MRI scans.

\section{ACKNOWLEDGEMENT}

The corresponding author would like to thank John Kender from Computer Science Department, Columbia University, for the helpful discussion. Authors would also like to thank Anthony L. D'Ambrosio from neurosurgery department for providing the clinical data. The funding source of this project comes from Department of 
neurosurgery, Columbia University.

\section{REFERENCES}

[1] Atam P.Dhawan, "Image registration", book chapter in "Medical Image Analysis", p254-p259, published by John Wiley \& Sons, 2003

[2] Sylvain Prima, Sébastien Ourselin, and Nicholas Ayache, "Computation of the Mid-Sagittal Plane In 3-D Brain Images: IEEE transaction on medical imaging, vol.21, no.2, february 2002

[3] Celina Imielinska, Xin Liu, Joel Rosiene et al., "Towards Objective Quantification of Perfusion-Weighted Computed Tomography in the Setting of Subarachnoid Hemorrhage: Quantification of Symmetry and Automated Delineation of Vascular Territories " ,Journal of Academic Radiology ,2005

[4] M. E. Brummer, "Hough transform detection of the longitudinal fissure in tomographic head images," IEEE Trans. Med. Imag., vol. 10, pp. 74-81, Mar. 1991.

[5] B. A. Ardekani, J. Kershaw, M. Braun, and I. Kanno, "Automatic detection of the mid-sagittal plane in 3-D brain images," IEEE Trans. Med Imag., vol. 16, pp. 947-952, Dec. 1997.

[6] C. Sun and J. Sherrah, "3D symmetry detection using the extended gaussian image," IEEE Trans. Pattern Anal. Machine Intell., vol. 19, pp. 164-168, Feb 1997

[7] Y. Liu, R. T. Collins, and W. E. Rothfus, "Robust Midsagittal Plane Extraction from Normal and Pathological 3D Neuroradiology Images" IEEE Transactions on Medical Imaging, Vol. 20, No. 3, March, 2001, pp. 175 - 192.

[8] P. Minovic , S. Ishikawa, K. Kato, "Symmetry Identification of a 3-D Object Represented by Octree", IEEE Transactions on Pattern Analysis and Machine Intelligence, v.15 n.5, p.507-514, May 1993

[9] P. C. Marais, R. Guillemaud, M. Sakuma, A. Zisserman, and M. Brady,"Visualising cerebral asymmetry," in Lecture Notes in Computer Science K. H. Höhne and R. Kikinis, Eds, Hamburg, Germany: Springer,Sept. 1996, vol 